OPEN ACCESS

Edited by:

Jean-Paul Deslypere,

Besins Healthcare, Thailand

Reviewed by:

Robert L. Lins,

Retired, Antwerpen, Belgium

Domenico Criscuolo,

Genovax S.r.l., Italy

${ }^{*}$ Correspondence:

Hsien-Feng Lin

sllin6@yahoo.com.tw

Specialty section:

This article was submitted to Pharmaceutical Medicine and Outcomes Research,

a section of the journal

Frontiers in Pharmacology

Received: 08 October 2017 Accepted: 09 November 2017 Published: 22 November 2017

Citation:

Lai S-W, Liao K-F, Lin C-L and Lin H-F (2017) Dipeptidyl Peptidase-4 Inhibitors Use and Relative Risk of Ischemic Cerebrovascular Disease in Type 2 Diabetic Patients in a Case-Control Study.

Front. Pharmacol. 8:859. doi: 10.3389/fphar.2017.00859

\section{Dipeptidyl Peptidase-4 Inhibitors Use and Relative Risk of Ischemic Cerebrovascular Disease in Type 2 Diabetic Patients in a Case-Control Study}

\author{
Shih-Wei Lai ${ }^{1,2}$, Kuan-Fu Liao ${ }^{3,4}$, Cheng-Li Lin ${ }^{1,5}$ and Hsien-Feng Lin ${ }^{2,6 *}$ \\ ' Department of Medicine, China Medical University, Taichung, Taiwan, ${ }^{2}$ Department of Family Medicine, China Medical \\ University Hospital, Taichung, Taiwan, ${ }^{3}$ Department of Medicine, Tzu Chi University, Hualien, Taiwan, ${ }^{4}$ Department of Internal \\ Medicine, Taichung Tzu Chi General Hospital, Taichung, Taiwan, ${ }^{5}$ Management Office for Health Data, China Medical \\ University Hospital, Taichung, Taiwan, ${ }^{6}$ Department of Chinese Medicine, China Medical University, Taichung, Taiwan
}

Background and Objectives: Limited research focuses on the risk of ischemic cerebrovascular disease associated with use of dipeptidyl peptidase-4 inhibitors (DPP4 inhibitors) in patients with type 2 diabetes mellitus in Taiwan. This study aimed to investigate the association between DPP-4 inhibitors use and the first episode of ischemic cerebrovascular disease.

Methods: We designed a case-control study using the database of the Taiwan National Health Insurance Program. There were 1999 type 2 diabetic subjects aged 20-84 years with the first episode of ischemic cerebrovascular disease from 2000 to 2013 as the cases, and 7996 sex- and age-matched, randomly selected type 2 diabetic subjects aged 20-84 years without any type of cerebrovascular diseases as the matched controls. We estimated the odds ratio (OR) and 95\% confidence interval (Cl) of ischemic cerebrovascular disease associated with cumulative duration of DPP-4 inhibitors use by the multivariable logistic regression model.

Results: After adjustment for confounding variables, the adjusted OR of ischemic cerebrovascular disease was $0.96(95 \% \mathrm{Cl} 0.95,0.97)$ in subjects with ever use of DPP4 inhibitors as increase in use duration for every 1 month, compared with never use. The sub-analysis disclosed that the adjusted ORs of ischemic cerebrovascular disease were 1.57 (95\% Cl 1.36, 1.80) for subjects with cumulative duration of DPP-4 inhibitors use $<1$ year, and $0.70(95 \% \mathrm{Cl} 0.57,0.87)$ for subjects with cumulative duration of DPP-4 inhibitors use $\geq 1$ year, compared with never use.

Conclusion: Our findings suggest that DPP-4 inhibitors use correlates with relative risk reduction of the first episode of ischemic cerebrovascular disease in type 2 diabetic patients in a duration-dependent response. The beneficial effect will be marked when DPP-4 inhibitors use is $\geq 1$ year.

Keywords: diabetes mellitus, DPP-4 inhibitors, ischemic cerebrovascular disease, National Health Insurance Program, Taiwan 


\section{INTRODUCTION}

Dipeptidyl peptidase-4 inhibitors (DPP-4 inhibitors) are classified as a new oral anti-diabetic medication and are widely used for management of type 2 diabetes mellitus (Baetta and Corsini, 2011). In addition to their glucose-lowering effects, current evidence discloses that DPP-4 inhibitors have beneficial effects on the major adverse cardiovascular events (Monami et al., 2011; Scheen, 2013; Davidson, 2014; Kaneko and Narukawa, 2016).

Cerebrovascular disease ranked the fourth leading cause of death and diabetes mellitus ranked the fifth leading cause of death in Taiwan in 2016, respectively (Ministry of Health and Welfare, 2017a). To date, conclusive results on the risk of ischemic cerebrovascular disease associated with DPP-4 inhibitors use in Taiwan are limited. If the association between the risk of ischemic cerebrovascular disease and DPP-4 inhibitors use substantially exists, the drug of choice for management of type 2 diabetes mellitus can be suggested in Taiwan. Therefore, we designed a case-control study using the database of the Taiwan National Health Insurance Program to investigate the association between DPP-4 inhibitors use and the first episode of ischemic cerebrovascular disease.

\section{MATERIALS AND METHODS}

\section{Study Design and Study Population}

The methodology was adapted from previous studies (Lai et al., 2016, 2017j; Liao et al., 2017b). It did not need to write published protocol details. We summarized them as follows and cited the relevant references. We designed a case-control study using the database of the Taiwan National Health Insurance Program. Taiwan is an independent country with more than 23 million persons (Chan et al., 2016; Chang and Yu, 2016; Chang et al., 2016; Chen and Wu, 2016; Chen S.Y. et al., 2016; Chen Y.F. et al., 2016; Hsieh et al., 2016; Hsu and Yin, 2016; Huang and Chang, 2016; Lin and Lin, 2016; Maa and Leu, 2016; Ooi, 2016; Yu et al., 2016; Lai et al., 2017g; Lee et al., 2017; Liang et al., 2017; Liao et al., 2017a,c; Lin et al., 2017a; Liu et al., 2017; Wen and Yin, 2017; Wu et al., 2017; Yang J.S. et al., 2017; Yang M.D. et al., 2017). The program launched in March 1995, and has covered nearly 99.6\% of 23 million persons at the end of 2015 (Ministry of Health and Welfare, 2017b). The program details have been described in previous studies (Lai et al., 2010; Chen H.Y. et al., 2016; Tsai et al., 2016; Chu et al., 2017). The Research Ethics Committee of China Medical University and Hospital in Taiwan approved the study (CMUH-104-REC2-115).

\section{Inclusion Criteria}

We selected type 2 diabetic subjects aged 20-84 years with the first episode of ischemic cerebrovascular disease as the cases during the period of 2000-2013 based on International Classification of Diseases, 9th Revision, Clinical Modification (ICD-9 codes 433, 434, and 435). The date of a subject being diagnosed with the first episode of ischemic cerebrovascular disease was defined as the index date. Additionally, for every one case with ischemic cerebrovascular disease, approximately four type 2 diabetic subjects aged 20-84 years without any type of cerebrovascular diseases were randomly selected as the matched controls. The cases and the matched controls were matched with sex, age (5-year interval), comorbidities, and the year of index date.

\section{Selection of Comorbidities}

Comorbidities potentially related to ischemic cerebrovascular disease before index date were identified as follows: alcoholrelated disease, atrial fibrillation, chronic kidney disease, chronic obstructive pulmonary disease, coronary artery disease, hyperlipidemia, and hypertension. Based on the ICD-9 codes, the diagnosis validity of comorbidities was discussed in previous studies (Lai et al., 2017a,b,c,d,e,f; Liao et al., 2017e; Lin et al., $2017 b, c)$.

\section{Definition of DPP-4 Inhibitors Use and Other Anti-diabetic Medications Use}

Prescription histories of medications studied were collected. Other anti-diabetic medications on Taiwan market during 20002013 were metformin, sulfonylureas, $\alpha$-glucosidase inhibitors, thiazolidinediones, and insulins. The definition of medications use was adapted from previous studies (Cheng et al., 2017; Lai et al., 2017h,i; Liao et al., 2017d,f). Briefly, subjects with at least a prescription for medications studied before index date were classified into "ever use." Subjects without a prescription of medications studied before index date were classified into "never use."

\section{Statistical Analysis}

At first, we made a comparison of sex, age, medications, and comorbidities between the cases and the matched controls by using the Chi-square test for categorized variables and the $t$-test for continuous variables. Then, variables which were significantly associated with ischemic cerebrovascular disease in the univariable logistic regression model were further tested by the multivariable logistic regression model. We estimated the odds ratio (OR) and 95\% confidence interval (CI) for the relative risk of ischemic cerebrovascular disease associated with cumulative duration of DPP-4 inhibitors use. The probability value $<0.05$ was considered statistically significant (SAS software version 9.2, SAS Institute, Inc., Cary, NC, United States).

\section{RESULTS}

\section{Basic Characteristics of the Study Population}

Table 1 discloses the basic characteristics of the study population. There were 1999 cases with the first episode of ischemic cerebrovascular disease in 2000-2013 and 7996 matched controls without any type of cerebrovascular diseases, with similar distributions of sex and age. The mean ages (standard deviation) were 67.8 (10.6) years in cases and $67.7(10.6)$ years in matched controls, without statistical significance $(t$-test, $P=0.67)$. The 
cases were more likely to have higher proportions of ever use of DPP-4 inhibitors, ever use of other anti-diabetic medications, alcohol-related disease, atrial fibrillation, chronic kidney disease, coronary artery disease, hyperlipidemia, and hypertension than the matched controls, with statistical significance (Chi-square test, $P<0.05$, for all).

\section{Relative Risk of Ischemic Cerebrovascular Disease Associated with Cumulative Duration of DPP-4 Inhibitors Use}

After adjustment for confounding variables including other antidiabetic medications, alcohol-related disease, atrial fibrillation, chronic kidney disease, chronic obstructive pulmonary disease, coronary artery disease, hyperlipidemia, and hypertension, the adjusted OR of ischemic cerebrovascular disease was 0.96 (95\% CI $0.95,0.97$ ) in subjects with ever use of DPP-4 inhibitors as increase in use duration for every 1 month, compared with never use. The sub-analysis disclosed that the adjusted ORs of ischemic cerebrovascular disease were 1.57 (95\% CI 1.36, 1.80) for subjects with cumulative duration of DPP-4 inhibitors use $<1$ year, and 0.70 (95\% CI $0.57,0.87$ ) for subjects with cumulative duration of DPP-4 inhibitors use $\geq 1$ year, compared with never use (Table 2).

\section{DISCUSSION}

In this case-control study, we found that DPP-4 inhibitors use was associated with decreased odds of the first episode of ischemic cerebrovascular disease in type 2 diabetic patients in a

TABLE 1 | Basic characteristics between cases with ischemic cerebrovascular disease and matched controls.

\begin{tabular}{|c|c|c|c|c|c|}
\hline \multirow[b]{2}{*}{ Variable } & \multicolumn{2}{|c|}{$\begin{array}{l}\text { Matched controls } \\
\qquad N=7996\end{array}$} & \multicolumn{2}{|c|}{$\begin{array}{l}\text { Cases with ischemic cerebrovascular } \\
\text { disease } N=1999\end{array}$} & \multirow[b]{2}{*}{$P$-value* } \\
\hline & $N$ & (\%) & $N$ & $(\%)$ & \\
\hline \multicolumn{5}{|l|}{ Sex } & \multirow[t]{3}{*}{0.99} \\
\hline Female & 3580 & $(44.8)$ & 895 & $(44.8)$ & \\
\hline Male & 4416 & $(55.2)$ & 1104 & $(55.2)$ & \\
\hline $50-64$ & 2500 & $(31.3)$ & 625 & $(31.3)$ & \multirow{2}{*}{0.99} \\
\hline $65-84$ & 5000 & (62.5) & 1250 & $(62.5)$ & \\
\hline Age (years), mean \pm standard deviation ${ }^{\dagger}$ & \multicolumn{2}{|c|}{$67.7 \pm 10.6$} & \multicolumn{2}{|c|}{$67.8 \pm 10.6$} & 0.67 \\
\hline Ever use of DPP-4 inhibitors & 1485 & (18.6) & 446 & $(22.3)$ & $<0.001$ \\
\hline Atrial fibrillation & 220 & (2.75) & 158 & $(7.90)$ & $<0.001$ \\
\hline Chronic kidney disease & 1602 & (20.0) & 441 & $(22.1)$ & 0.04 \\
\hline Chronic obstructive pulmonary disease & 2617 & (32.7) & 597 & $(29.9)$ & 0.01 \\
\hline Coronary artery disease & 3586 & (44.9) & 948 & $(47.4)$ & 0.04 \\
\hline Hyperlipidemia & 5697 & (71.3) & 1480 & $(74.0)$ & 0.04 \\
\hline Hypertension & 6517 & (81.5) & 1830 & $(91.6)$ & $<0.001$ \\
\hline
\end{tabular}

Data are presented as the number of subjects in each group with percentages given in parentheses. ${ }^{*}$ Chi-square test and ${ }^{\dagger} t$-test comparing subjects with and without ischemic cerebrovascular disease.

TABLE 2 | Relative risk of ischemic cerebrovascular disease associated with cumulative duration of DPP-4 inhibitors use.

\begin{tabular}{|c|c|c|c|c|c|}
\hline Variable & Case number/control number & Crude OR & $(95 \% \mathrm{Cl})$ & Adjusted OR ${ }^{\dagger}$ & $(95 \% \mathrm{Cl})$ \\
\hline Never use of DPP-4 inhibitors as a reference & $1553 / 6511$ & 1.00 & (Reference) & 1.00 & (Reference) \\
\hline $\begin{array}{l}\text { Cumulative duration of DPP- } 4 \text { inhibitors use } \\
\text { (increase in use duration for every } 1 \text { month) }\end{array}$ & $446 / 1485$ & 0.96 & $(0.95,0.98)$ & 0.96 & $(0.95,0.97)$ \\
\hline \multicolumn{6}{|l|}{ Cumulative duration of DPP-4 inhibitors use } \\
\hline$<1$ year & $340 / 880$ & 1.62 & $(1.41,1.86)$ & 1.57 & $(1.36,1.80)$ \\
\hline$\geq 1$ year & $106 / 605$ & 0.74 & $(0.59,0.91)$ & 0.70 & $(0.57,0.87)$ \\
\hline
\end{tabular}

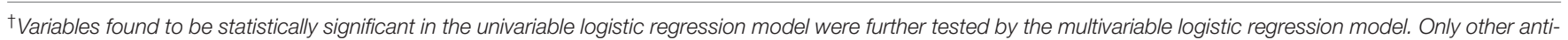

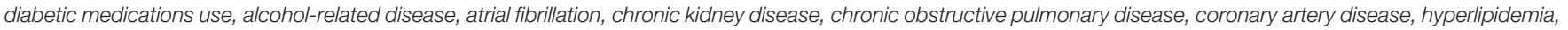
and hypertension could be further tested. 
duration-dependent response. The effect would be marked when DPP- 4 inhibitors use was $\geq 1$ year. This finding was consistent with previous studies disclosing that DPP-4 inhibitors use was associated with lower risk of ischemic cerebrovascular disease (Ou et al., 2015; Shih et al., 2016). We found that if DPP-4 inhibitors use was less than 1 year, the odds would be high (adjusted OR1.57). That is, short-term of DPP-4 inhibitors use does not have the beneficial effect. Clinicians should keep in mind about the risk of ischemic cerebrovascular disease during the first year of DPP-4 inhibitors use in type 2 diabetic patients. We suggest that only long-term of DPP-4 inhibitors use for 1 year or longer, type 2 diabetic patients can have the beneficial effect on risk reduction of ischemic cerebrovascular disease.

Hemoglobin A1c is an important indicator for long-term glycemic control. Previous studies disclosed that high levels of hemoglobin Alc were associated with increased risk of cerebrovascular disease (Goto et al., 2015; Cavender et al., 2016). Although DPP-4 inhibitors are not the drug of first choice for type 2 diabetes mellitus by the guidelines of American Diabetes Association (American Diabetes Association, 2017). DPP-4 inhibitors use still can reduce hemoglobin A1c by $0.5-1 \%$ (Madsbad et al., 2008; Grunberger, 2014). In our opinion, patients using other anti-diabetic medications but poor glycemic control would additionally use DPP-4 inhibitors. When initiating DPP4 inhibitors, Hemoglobin A1c levels of these patients should be high. The risk for ischemic cerebrovascular disease was still greater. Only using DPP-4 inhibitors for 1 year or longer and then hemoglobin A1c gradually reducing, these patients could have a chance to be in good glycemic control. Thus, the risk of ischemic cerebrovascular disease was further reduced. That at least partially explains why the odds of ischemic cerebrovascular disease would be high during the first year of DPP-4 inhibitors use, and then the odds would be reduced after 1 year.

Some limitations should be mentioned. First, due to the inherent limitation, hemoglobin Alc was not recorded in the database. Our study could not prove the association between the risk of ischemic cerebrovascular disease and hemoglobin Alc levels. Second, Table 1 discloses that about $97 \%$ of study subjects had ever used other anti-diabetic medications. It is difficult to investigate the absolute risk of ischemic cerebrovascular disease associated with DPP-4 inhibitors use alone due to the small eligible number for DPP-4 inhibitors use alone. The rational option was to investigate the relative risk of ischemic cerebrovascular disease associated with DPP-4 inhibitors use after adjustment for other anti-diabetic medications. Third, a case-control study could not prove the causal relationship. Further prospective cohort research is warranted to focus on the

\section{REFERENCES}

American Diabetes Association (2017). Pharmacologic approaches to glycemic treatment. Diabetes Care 40, S64-S74. doi: 10.2337/dc17-S011

Baetta, R., and Corsini, A. (2011). Pharmacology of dipeptidyl peptidase-4 inhibitors: similarities and differences. Drugs 71, 1441-1467. doi: 10.2165/ 11591400-000000000-00000

Cavender, M. A., Scirica, B. M., Raz, I., Steg, P. G., Mcguire, D. K., Leiter, L. A., et al. (2016). Cardiovascular outcomes of patients in SAVOR-TIMI 53 by baseline association between the absolute risk of ischemic cerebrovascular disease and DPP-4 inhibitors use alone.

Some strengths should be emphasized. The title clearly and precisely reflects the findings of the study. The statistical methods are used validate. The prior works are properly and fully cited. Immortal time bias can be minimized in a case-control study. The results are convincing.

\section{CONCLUSION}

Our findings suggest that DPP-4 inhibitors use correlates with relative risk reduction of the first episode of ischemic cerebrovascular disease in type 2 diabetic patients in a durationdependent response. The beneficial effect will be marked when DPP- 4 inhibitors use is $\geq 1$ year.

\section{ETHICS STATEMENT}

Insurance reimbursement claims data used in this study were available for public access. Patient identification numbers had been scrambled to ensure confidentiality. Patient informed consent was not required.

\section{AUTHOR CONTRIBUTIONS}

S-WL and K-FL contributed to the conception of the article, initiated the draft of the article, revised the article, and contributed equally to the article. C-LL conducted the data analysis and reviewed the article. H-FL participated in the data interpretation and revised the article.

\section{ACKNOWLEDGMENTS}

This study was supported in part by Taiwan Ministry of Health and Welfare Clinical Trial Center (MOHW106-TDU-B-212113004), China Medical University Hospital, Academia Sinica Taiwan Biobank Stroke Biosignature Project (BM10601010036), Taiwan Clinical Trial Consortium for Stroke (MOST 106-2321-B039-005), Tseng-Lien Lin Foundation, Taichung, Taiwan, Taiwan Brain Disease Foundation, Taipei, Taiwan, and Katsuzo and Kiyo Aoshima Memorial Funds, Japan. These funding agencies did not influence the study design, data collection and analysis, decision to publish, or preparation of the manuscript.

hemoglobin A1c. Am. J. Med. 129, 340:e1-8. doi: 10.1016/j.amjmed.2015. 09.022

Chan, C. Y., Lien, C. H., Lee, M. F., and Huang, C. Y. (2016). Quercetin suppresses cellular migration and invasion in human head and neck squamous cell carcinoma (HNSCC). Biomedicine 6, 15. doi: 10.7603/s40681-016-0015-3

Chang, L. C., and Yu, Y. L. (2016). Dietary components as epigenetic-regulating agents against cancer. Biomedicine 6, 2. doi: 10.7603/s40681-016-0002-8

Chang, W. S., Liu, L. C., Hsiao, C. L., Su, C. H., Wang, H. C., Ji, H. X., et al. (2016). The contributions of the tissue inhibitor of metalloproteinase- 1 genotypes to 
triple negative breast cancer risk. Biomedicine 6, 4. doi: 10.7603/s40681-0160004-6

Chen, H. F., and Wu, K. J. (2016). Epigenetics, TET proteins, and hypoxia in epithelial-mesenchymal transition and tumorigenesis. Biomedicine 6, 1 . doi: 10.7603/s40681-016-0001-9

Chen, H. Y., Lin, C. L., Lai, S. W., and Kao, C. H. (2016). Association of selective serotonin reuptake inhibitor use and acute angle-closure glaucoma. J. Clin. Psychiatry 77, e692-6. doi: 10.4088/JCP.15m10038

Chen, S. Y., Hsu, Y. M., Lin, Y. J., Huang, Y. C., Chen, C. J., Lin, W. D., et al. (2016). Current concepts regarding developmental mechanisms in diabetic retinopathy in Taiwan. Biomedicine 6, 7. doi: 10.7603/s40681-016-0007-3

Chen, Y. F., Wu, K. J., Huang, W. S., Hsieh, Y. W., Wang, Y. W., Tsai, H. Y., et al. (2016). Neuroprotection of Gueichih-Fuling-Wan on cerebral ischemia/reperfusion injury in streptozotocin-induced hyperglycemic rats via the inhibition of the cellular apoptosis pathway and neuroinflammation. Biomedicine 6, 21. doi: 10.7603/s40681-016-0021-5

Cheng, K. C., Liao, K. F., Lin, C. L., and Lai, S. W. (2017). Correlation of proton pump inhibitors with pulmonary tuberculosis: a case-control study in Taiwan. Front. Pharmacol. 8:481. doi: 10.3389/fphar.2017.00481

Chu, C. S., Lin, C. C., Peng, C. Y., Chuang, P. H., Su, W. P., Lai, S. W., et al. (2017). Does pyogenic liver abscess increase the risk of delayed-onset primary liver cancer?: evidence from a nationwide cohort study. Medicine 96:e7785. doi: 10.1097/MD.0000000000007785

Davidson, M. H. (2014). Potential impact of dipeptidyl peptidase-4 inhibitors on cardiovascular pathophysiology in type 2 diabetes mellitus. Postgrad. Med. 126, 56-65. doi: 10.3810/pgm.2014.05.2756

Goto, A., Noda, M., Matsushita, Y., Goto, M., Kato, M., Isogawa, A., et al. (2015). Hemoglobin a1c levels and the risk of cardiovascular disease in people without known diabetes: a population-based cohort study in Japan. Medicine 94:e785. doi: 10.1097/MD.0000000000000785

Grunberger, G. (2014). Clinical utility of dipeptidyl peptidase-4 inhibitors: a descriptive summary of current efficacy trials. Eur. J. Clin. Pharmacol. 70, 1277-1289. doi: 10.1007/s00228-014-1727-5

Hsieh, T. C., Wu, Y. C., Sun, S. S., Yen, K. Y., and Kao, C. H. (2016). Treating hepatocellular carcinoma with $90 \mathrm{Y}$-bearing microspheres: a review. Biomedicine 6, 19. doi: 10.7603/s40681-016-0019-z

Hsu, Y. M., and Yin, M. C. (2016). EPA or DHA enhanced oxidative stress and aging protein expression in brain of d-galactose treated mice. Biomedicine 6, 17. doi: 10.7603/s40681-016-0017-1

Huang, Y. P., and Chang, N. W. (2016). PPARalpha modulates gene expression profiles of mitochondrial energy metabolism in oral tumorigenesis. Biomedicine 6, 3. doi: 10.7603/s40681-016-0003-7

Kaneko, M., and Narukawa, M. (2016). Meta-analysis of dipeptidyl peptidase4 inhibitors use and cardiovascular risk in patients with type 2 diabetes mellitus. Diabetes Res. Clin. Pract. 116, 171-182. doi: 10.1016/j.diabres.2016. 04.012

Lai, S. W., Liao, K. F., Liao, C. C., Muo, C. H., Liu, C. S., and Sung, F. C. (2010). Polypharmacy correlates with increased risk for hip fracture in the elderly: a population-based study. Medicine 89, 295-299. doi: 10.1097/MD. 0b013e3181f15efc

Lai, S. W., Lin, H. F., Lin, C. L., and Liao, K. F. (2016). Long-term effects of pioglitazone on first attack of ischemic cerebrovascular disease in older people with type 2 diabetes: a case-control study in Taiwan. Medicine 95:e4455. doi: 10.1097/MD.0000000000004455

Lai, S. W., Lin, C. H., Lin, H. F., Lin, C. L., Lin, C. C., and Liao, K. F. (2017a). Herpes zoster correlates with increased risk of Parkinson's disease in older people: a population-based cohort study in Taiwan. Medicine 96:e6075. doi: 10.1097/MD.0000000000006075

Lai, S. W., Lin, C. L., and Liao, K. F. (2017b). Glaucoma correlates with increased risk of Parkinson's disease in the elderly: a national-based cohort study in Taiwan. Curr. Med. Res. Opin. 33, 1511-1516. doi: 10.1080/03007995.2017. 1322570

Lai, S. W., Lin, C. L., and Liao, K. F. (2017c). Glaucoma may be a non-memory manifestation of Alzheimer's disease in older people. Int. Psychogeriatr. 29, 1535-1541. doi: 10.1017/S1041610217000801

Lai, S. W., Lin, C. L., and Liao, K. F. (2017d). Head and neck cancer associated with increased rate of pulmonary tuberculosis in a population-based cohort study. Medicine 96:e8366. doi: 10.1097/MD.0000000000008366
Lai, S. W., Lin, C. L., and Liao, K. F. (2017e). Population-based cohort study investigating the correlation of diabetes mellitus with pleural empyema in adults in Taiwan. Medicine 96:e7763. doi: 10.1097/MD.0000000000007763

Lai, S. W., Lin, C. L., and Liao, K. F. (2017f). Predialysis chronic kidney disease correlates with increased risk of pyogenic liver abscess: a population-based cohort study. Eur. J. Clin. Invest. 47, 694-701. doi: 10.1111/eci.12793

Lai, S. W., Lin, C. L., and Liao, K. F. (2017g). Risk of contracting pneumonia among patients with predialysis chronic kidney disease: a population-based cohort study in Taiwan. Biomedicine 7:20. doi: 10.1051/bmdcn/2017070320

Lai, S. W., Lin, C. L., and Liao, K. F. (2017h). Tamoxifen use correlates with increased risk of the first episode of ischemic cerebrovascular disease in older women with breast cancer: a case-control study in Taiwan. Front. Pharmacol. 8:742. doi: 10.3389/fphar.2017.00742

Lai, S. W., Lin, C. L., and Liao, K. F. (2017i). Use of oral corticosteroids and risk of hip fracture in the elderly in a case-control study. Front. Pharmacol. 8:625. doi: 10.3389/fphar.2017.00625

Lai, S.-W., Lin, H.-F., Lin, C.-L., and Liao, K.-F. (2017j). No association between losartan use and acute pancreatitis in hypertensive patients. Eur. J. Hosp. Pharm. 24, 120-123.

Lee, M. R., Lin, C., Lu, C. C., Kuo, S. C., Tsao, J. W., Juan, Y. N., et al. (2017). YC-1 induces G0/G1 phase arrest and mitochondria-dependent apoptosis in cisplatin-resistant human oral cancer CAR cells. Biomedicine 7, 12. doi: 10.1051/ bmdcn/2017070205

Liang, Y. C., Hu, J. C., Li, P. Y., Huang, G. J., Kuo, Y. H., and Chao, C. Y. (2017). Torenia concolor Lindley var. formosana Yamazaki extracts improve inflammatory response and lipid accumulation via PPARs activation. Biomedicine 7:18. doi: 10.1051/bmdcn/2017070318

Liao, C. F., Yang, T. Y., Chen, Y. H., Yao, C. H., Way, T. D., and Chen, Y. S. (2017a). Effects of swimming exercise on nerve regeneration in a rat sciatic nerve transection model. Biomedicine 7:3. doi: 10.1051/bmdcn/2017070103

Liao, K. F., Cheng, K. C., Lin, C. L., and Lai, S. W. (2017b). Etodolac and the risk of acute pancreatitis. Biomedicine 7:4. doi: 10.1051/bmdcn/2017070104

Liao, K. F., Huang, P. T., Lin, C. C., Lin, C. L., and Lai, S. W. (2017c). Fluvastatin use and risk of acute pancreatitis:a population-based case-control study in Taiwan. Biomedicine 7:17. doi: 10.1051/bmdcn/2017070317

Liao, K. F., Lin, C. L., and Lai, S. W. (2017d). Nationwide case-control study examining the association between tamoxifen use and Alzheimer's disease in aged women with breast cancer in Taiwan. Front. Pharmacol. 8:612. doi: 10.3389/fphar.2017.00612

Liao, K.-F., Lin, C.-L., and Lai, S.-W. (2017e). Parkinson's disease and risk of colorectal cancer: a population-based case-control study in Taiwan. Neurology Asia 22, 133-138.

Liao, K.-F., Lin, C.-L., and Lai, S.-W. (2017f). Population-based case-control study assessing the association between statins use and pulmonary tuberculosis in Taiwan. Frontiers in Pharmacology 8:597. doi: 10.3389/fphar.2017.00597

Lin, C. H., Lin, W. C., and Chang, J. S. (2017a). Presentations and management of different causes of chylothorax in children: one medical center's experience. Biomedicine 7:5. doi: 10.1051/bmdcn/2017070105

Lin, H.-F., Liao, K.-F., Chang, C.-M., Lin, C.-L., and Lai, S.-W. (2017b). Populationbased cohort study examining the association between splenectomy and empyema in adults in Taiwan. BMJ Open 7:e015101. doi: 10.1136/bmjopen2016-015101

Lin, H. F., Liao, K. F., Chang, C. M., Lin, C. L., Lin, C. H., and Lai, S. W. (2017c). Use of thiazolidinediones and risk of hip fracture in old people in a case-control study in Taiwan. Medicine 96:e7712. doi: 10.1097/MD.0000000000007712

Lin, W. C., and Lin, C. H. (2016). Multidetector computed tomography in the evaluation of pediatric acute abdominal pain in the emergency department. Biomedicine 6, 10. doi: 10.7603/s40681-016-0010-8

Liu, Y. L., Liu, J. H., Wang, I. K., Ju, S. W., Yu, T. M., Chen, I. R., et al. (2017). Association of inflammatory cytokines with mortality in peritoneal dialysis patients. Biomedicine 7:1. doi: 10.1051/bmdcn/2017070101

Maa, M. C., and Leu, T. H. (2016). Src is required for migration, phagocytosis, and interferon beta production in Toll-like receptor-engaged macrophages. Biomedicine 6:14. doi: 10.7603/s40681-016-0014-4

Madsbad, S., Krarup, T., Deacon, C. F., and Holst, J. J. (2008). Glucagon-like peptide receptor agonists and dipeptidyl peptidase- 4 inhibitors in the treatment of diabetes: a review of clinical trials. Curr. Opin. Clin. Nutr. Metab. Care 11, 491-499. doi: 10.1097/MCO.0b013e328302f414 
Ministry of Health and Welfare (2017a). Statistics of Causes of Death. Available at: http://www.mohw.gov.tw/EN/Ministry/Index.aspx

Ministry of Health and Welfare (2017b). Taiwan Health and Welfare Report. Available at: http://www.mohw.gov.tw

Monami, M., Dicembrini, I., Martelli, D., and Mannucci, E. (2011). Safety of dipeptidyl peptidase-4 inhibitors: a meta-analysis of randomized clinical trials. Curr. Med. Res. Opin. 27(Suppl. 3), 57-64. doi: 10.1185/03007995.2011. 602964

Ooi, H. (2016). Bedside pleuroscopy in Taiwan: a great vision for critically-ill patients and intensivists. Biomedicine 6, 13. doi: 10.7603/s40681-016-0013-5

Ou, S. M., Shih, C. J., Chao, P. W., Chu, H., Kuo, S. C., Lee, Y. J., et al. (2015). Effects on clinical outcomes of adding dipeptidyl peptidase-4 inhibitors versus sulfonylureas to metformin therapy in patients with type 2 diabetes mellitus. Ann. Intern. Med. 163, 663-672. doi: 10.7326/M150308

Scheen, A. J. (2013). Cardiovascular effects of dipeptidyl peptidase-4 inhibitors: from risk factors to clinical outcomes. Postgrad. Med. 125, 7-20. doi: 10.3810/ pgm.2013.05.2659

Shih, C. J., Chen, H. T., Kuo, S. C., Ou, S. M., and Chen, Y. T. (2016). Cardiovascular outcomes of dipeptidyl peptidase-4 inhibitors in elderly patients with type 2 diabetes: a nationwide study. J. Am. Med. Dir. Assoc. 17, 59-64. doi: 10.1016/j.jamda.2015.10.009

Tsai, T. Y., Lin, C. C., Peng, C. Y., Huang, W. H., Su, W. P., Lai, S. W., et al. (2016). The association between biliary tract inflammation and risk of digestive system cancers: a population-based cohort study. Medicine 95:e4427. doi: 10.1097/MD. 0000000000004427
Wen, Y. J., and Yin, M. C. (2017). The anti-inflammatory and anti-glycative effects of rosmarinic acid in the livers of type 1 diabetic mice. Biomedicine 7:19. doi: 10.1051/bmdcn/2017070319

Wu, M. H., Lee, T. H., Lee, H. P., Li, T. M., Lee, I. T., Shieh, P. C., et al. (2017). Kuei-Lu-Er-Xian-Jiao extract enhances BMP-2 production in osteoblasts. Biomedicine 7:2. doi: 10.1051/bmdcn/2017070102

Yang, J. S., Lu, C. C., Kuo, S. C., Hsu, Y. M., Tsai, S. C., Chen, S. Y., et al. (2017). Autophagy and its link to type II diabetes mellitus. Biomedicine 7, 8 . doi: 10.1051/bmdcn/2017070201

Yang, M. D., Lin, K. C., Lu, M. C., Jeng, L. B., Hsiao, C. L., Yueh, T. C., et al. (2017). Contribution of matrix metalloproteinases-1 genotypes to gastric cancer susceptibility in Taiwan. Biomedicine 7, 10. doi: 10.1051/bmdcn/2017070203

Yu, C. C., Chien, C. T., and Chang, T. C. (2016). M2 macrophage polarization modulates epithelial-mesenchymal transition in cisplatin-induced tubulointerstitial fibrosis. Biomedicine 6:5. doi: 10.7603/s40681-016-0005-5

Conflict of Interest Statement: The authors declare that the research was conducted in the absence of any commercial or financial relationships that could be construed as a potential conflict of interest.

Copyright (c) 2017 Lai, Liao, Lin and Lin. This is an open-access article distributed under the terms of the Creative Commons Attribution License (CC BY). The use, distribution or reproduction in other forums is permitted, provided the original author(s) or licensor are credited and that the original publication in this journal is cited, in accordance with accepted academic practice. No use, distribution or reproduction is permitted which does not comply with these terms. 